\title{
Neurocognitive Disorder as a Family Illness
}

\section{Claudia lacobacci*}

Cognitive Neuroscience and Cerebellum Laboratory, Italy

\section{Neurocognitive Disorder}

Becoming a caregiver for members of their own family with neurocognitive disorder or dementia like Alzheimer's disease, frontotemporal dementia or dementia with Lewy bodies means take a new and unexpected role.

A caregiver can be defined as an unpaid person who helps another individual who has a chronic physical or psychological impairment and takes responsibility for him.

Typical duties of a caregiver might include managing medications or talking to doctors on someone's behalf; helping to bathe or dress someone; or taking care of household chores, meals, or bills for someone who cannot do these things alone. Becoming a caregiver for members of their own family means to take care of an adult person which cares of us in every moment.

We thought that the neurocognitive disorders were characteristic of aging and people over 65 years of age would have more risk to be affected by them. Instead today more people younger than 65 years receive a diagnosis of dementia (early-onset dementia or EOD). EOD is often misdiagnosed or its diagnosis is delayed because it has a more varied differential diagnosis than late-onset dementia. In case of EOD, often, the first symptoms are changes in personality or mood, confused with a mood disorder. For this reason, for the specialist get accurate diagnosis and treatment promptly are more difficult. EOD affects individuals at the height of their career and productivity and produces devastating consequences and financial loss for the patient's family as well as society [1].

It is heartbreaking and painful to watch an important member of family lose his attitudes and his cognitive capacities. For example, it is heartbreaking to see a mother lose their ability to care for her house and to go grocery shopping. It is painful to see a mother that can't cook anymore. It is heartbreaking to see a father that can't no longer walk the dog or go to the bank. In this case the major role of mother and father, or husband or wife can be lost within the family. The loved one can lose the ability to take care of himself and of the other members of family.

The neurocognitive disorder, when diagnosed, distresses all members of the family not only the person affected but all members of the family. The family members have to change their role becoming a caregiver in addition to being a son, a daughter, a father, a wife and a husband. The role of caregiver is an unexpected, undesirable, underappreciated role. No one could have expected that the loved one lost his abilities, so we are not prepared to become a caregiver. Moreover, when providing care for a loved one, caregivers can often neglect their own needs. This neglect, over time, can exact a heavy toll on the overall well-being of a caregiver. Became a caregiver means taking on a new role. It's a job.

It requires training and an attitude that allows you to take care of yourself as well as your own. It's not simple. Caregiving can affect the psychological and physical state, the relationship, the productivity of labor. Caregiving can lead to stress and can be dangerous for the caregiver. Prolonged periods of stress can lead to the situation called "burn-out". The burn-out is a severe condition that affects humor. A caregiver in "burn-out" can feel exhausted, listless, and unable to cope. This condition might affect the relation with loved one [2-4].

It's more difficult to become the caregiver of a young person. Sometimes we think that his difficulties are questions of will. We think that the abnormal behavior is spiteful of us. It's relatively easy to accept the difficulties in older people than in younger.

The caregivers passes through a lot of emotions. They may feel scared, frustrated, angry, depressed, resigned because it is difficult to accept the disease of the loved one. To prevent the burn-out of caregivers it is of vital importance to seek professional help. It's of vital importance to know as much as we can about the illness. A professional can try to teach the manner to support the loved one. It's important to be able to talk about our concerns. The caregiver must put his self to the level of the loved one. The worst attitude is to persevere in a behavior without realizing that the situation in the family has changed.

The education about the disease reveals to caregivers the ways in which these diseases gradually erode the person's ability to use normal intellectual potential, the difficulty to maintain emotional balance, the difficulty to plan and to carry out daily actions. Education can help the caregiver to communicate with the loved one when he is not able to do, to attract his attention, to understand the memory loss. We aren't born knowing how to communicate with a person with neurocognitive disease but we can learn. To learn we need a change of mood and acceptance. I think that the complete acceptance of the neurocognitive disease is very difficult for the family members.

Despite therapeutic journey in which the caregiver learns modalities to interact with the loved one, the acceptance is "incomplete". We learn to understand the disease and live together with. But every day we feel the loss in the heart.

In this case, we, psychologists play a very important role. We must be able to support the family members, we must listen to the concerns without judgment. We must be able to help the caregiver find the best way to interact with the loved one. We must help to face challenges and the emotions that change depending on the needs of the moment.

Initially the caregiver will get attention to the worries, to the changes in his life. In time, improving caregivers skills will help make caregiving less stressful and will likely improve the quality of the relationship with loved one.

The caregivers must learn to be empathic, patient, flexible and supportive.

*Corresponding author: lacobacci C, Cognitive Neuroscience and Cerebellum Laboratory, Italy, Tel: + 39 06515011; E-mail: cla.iacobacci@gmail.com

Received: July 12, 2017; Accepted: July 20, 2017; Published: July 27, 2017

Citation: Iacobacci C (2017) Neurocognitive Disorder as a Family Illness. Clin Exp Psychol 3: 158. doi: 10.4172/2471-2701.1000158

Copyright: (C) 2017 lacobacci C. This is an open-access article distributed under the terms of the Creative Commons Attribution License, which permits unrestricted use, distribution, and reproduction in any medium, provided the original author and source are credited. 
In fact, caregiver's awareness of the disease is crucial because it facilitates the understanding of the needs of the patient and therefore facilitates the care. With the right information, resources and tips on caregiving the caregiver can become expert at both caring for your charge and taking care of himself.

\section{References}

1. Fadil H, Borazanci A, Ait Ben Haddou E, Yahyaoui M, Korniychuk E, et al.
(2009) Early onset dementia. Int Rev Neurobiol 84: 245-62

2. Annerstedt L, Elmstahl S, Ingvad B, Samuelsson SM (2000) Family caregiving in dementia - An analysis of the caregiver's burden and the "breaking-point" when home care becomes inadequate. Scand J Public Health 28: 23-31.

3. Hepburn K, Lewis M, Tornatore J, Sherman CW, Dolloff J (2008) Caregiver's manual. Training program for assistance to people with dementia.

4. Wood FG (1991) The meaning of caregiving rehabilitation nursing. 